\title{
Häufige Symptome - seltene Differenzialdiagnose: Zervikale Lymphadenopathie, Fieber und unspezifisches Exanthem bei einem 8-jährigen Mädchen
}

Die Kikuchi-Lymphadenopathie (Kikuchi-Fujimoto Disease, KFD) ist eine benigne lymphohistiozytäre Erkrankung mit typischerweise zervikaler Lymphadenopatie und Fieber bislang unklarer Ätiologie. Vorgestellt wird der Fall eines 8-jährigen, vormals gesunden Mädchens, nicht-konsanguiner kaukasischer Eltern, die sich mit dieser seltenen Erkrankung präsentierte.

\section{Fallbericht}

\section{Anamnese}

Das Mädchen stellte sich vor mit einem Symptomkomplex aus rezidivierendem Antibiotika-resistentem Fieber, einer zervikalen Lymphadenopathie sowie einem Stamm-betonten polymorphen Exanthem.

Die Patientin hatte seit 12 Tagen rezidivierendes zweigipfliges Fieber über $40{ }^{\circ} \mathrm{C}$ und eine zervikale Lymphadenopathie. Am 5. Tag nach Beginn der Symptomatik war ein polymorphes Stamm-betontes Exanthem aufgetreten. Eine antibiotische Therapie mit Amoxicillin/Clavulansäure, die nach Beginn des Fiebers über 10 Tage verabreicht worden war, hatte keinen Effekt gezeigt. Einen Tag vor Beginn des Fiebers war die Patientin von einem 10-tägigen Auslandsaufenthalt aus Kreta zurückgekehrt.

\section{Klinischer Untersuchungsbefund}

Im klinischen Untersuchungsbefund bei Aufnahme zeigte sich ein 8-jähriges Mädchen in reduziertem Allgemeinzustand und gutem Ernährungszustand. Im pädiatrisch-internistischen Befund zeigte sich ein unspezifisches, Stamm-betontes, polymorphes Exanthem ( $\mathbf{A} \mathbf{b b} \mathbf{b} \mathbf{1})$. Weiterhin ließ sich eine bilaterale, vornehmlich rechtsseitige, zervikale Lymphadenopathie palpieren. Eine Hepatosplenomegalie fand sich nicht.

\section{Labor}

Laborchemisch lag eine Panzytopenie (Leukozyten $3,8 \times 10^{3} / \mu \mathrm{l}\left[4,3-11,4 \times 10^{3}\right]$, Hämoglobin von $8,7 \mathrm{~g} / \mathrm{dl}[10,6-13,2]$, Thrombozyten $\left.157 \times 10^{3} / \mu \mathrm{l}\left[199-367 \times 10^{3}\right]\right)$ mit unauffälligem Differenzialblutbild vor. Neben einer erhöhten LDH (530 U/I,

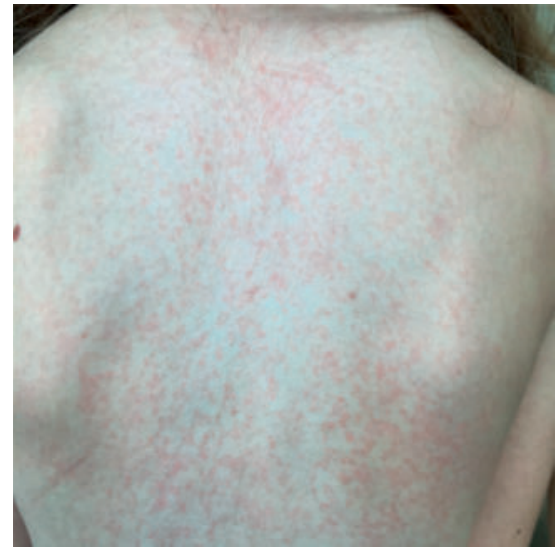

- Abb. 1 Polymorphes Stamm-betontes makulopapulöses Exanthem.

[<319]), zeigten sich deutlich erhöhte humorale Entzündungsmarker wie Serum-Amyloid A (432 mg/l, [<10]), C-reaktives Protein $(2,4 \mathrm{mg} / \mathrm{dl}[<0,5])$, Ferritin $(700 \mu \mathrm{g} / \mathrm{l},[7-84])$ und im Verlauf ein massiv erhöhtes Protein S100 A8/A9 (182560 ng/ $\mathrm{ml}[<2940])$ sowie Blutsenkungsgeschwindigkeit $(140 \mathrm{~mm} / 1 \mathrm{~h}[<20])$.

Die infektiologischen Differenzialdiagnosen (EBV, CMV, HSV, HIV, Mumps, Masern, Yersinien, Treponemen, Leishmanien, Tuberkulose, Bartonellen, Toxoplasmose, Brucellen, Coxiellen, Chlamydien; wegen des anamnestischen Auslandsaufenthaltes: Sandmücken-Fieber, West-Nil-Virus) wurden entsprechend der AWMF-Leitlinie Lymphknotenvergrößerung [1] serologisch ausgeschlossen. Blutkulturen blieben wiederholt ohne Erregernachweis.

\section{Diagnostik}

In der Sonografie ließen sich zervikal und supraklavikulär multiple vergrößerte (max. 4,1 cm) Lymphknoten mit ausgeprägter Umgebungsreaktion des Fettgewebes sowie Hyperperfusion darstellen ( $>$ Abb. 2). Weiterhin zeigten sich sonografisch eine Hepatosplenomegalie mit Leberund Milzvergrößerung um ca. $1 \mathrm{~cm}$ über dem altersentsprechenden Normbereich sowie ein minimaler Pleuraerguss linksseitig. Ein Röntgen-Thorax war unauffällig. Eine Echokardiografie erbrachte einen Normalbefund, insbesondere ohne Hinweis für
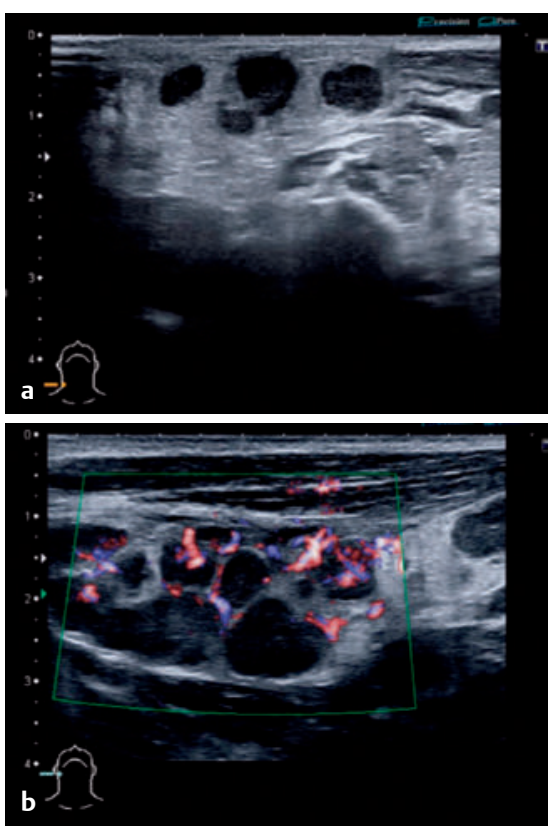

Abb. 2 Multiple, teils echoarme Lymphknoten mit deutlicher Hyperperfusion und ausgeprägter Umgebungsreaktion des angrenzenden Fettgewebes.

Veränderungen der Koronararterien oder einen Perikarderguss.

Zum Ausschluss einer hämatologischen Neoplasie erfolgten eine Knochenmarkpunktion und eine Lymphknotenexstirpation mit konsekutiver histopathologischer Aufarbeitung ( $\triangleright$ Abb. 3). Die Knochenmarkpunktion zeigte keine Hinweise für Malignität, Leishmanien oder Hämophagozytose.

\section{Diagnose und Verlauf}

Histopathologisch bot sich das Bild eines nekrotisch veränderten Lymphknotens mit flächigen Infiltraten in Parakortex und Pulpa, das - nach Ausschluss einer Leukämie bzw. Lymphoms - das typische Bild einer KFD aufwies ( $\triangleright$ Abb. 3). Eine wegen des persistierenden Fiebers und reduzierten Allgemeinzustandes initiierte Therapie mit hochdosiertem Methylprednisolon (30 mg/kg KG an 3 aufeinander folgenden Tagen), führte zu einer raschen und vollständigen Regredienz der Symptome. Die Patientin befindet sich seither in klinischer und laborchemischer Remission. 

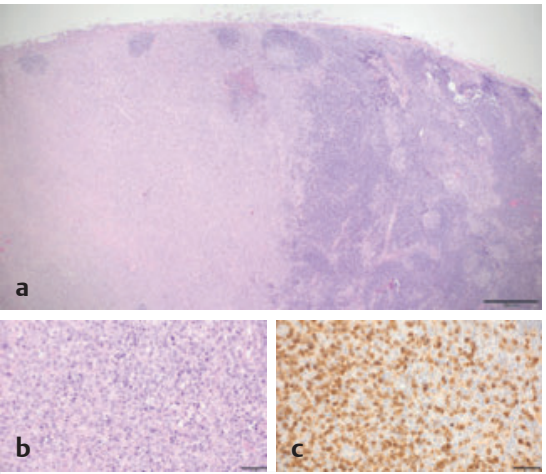

- Abb. 3 (a, HE-Färbung, Maßstab $500 \mu \mathrm{m})$ Flächige Infiltrate aus blastären Zellen in Parakortex und Pulpa; (b, HE-Färbung, Maßstab $50 \mu \mathrm{m}$ ) Zahlreiche, zentral nekrotisierende, Kerntrümmer; (c Myeoperoxidase-Färbung, Maßstab $50 \mu \mathrm{m})$ Myeloperoxidase-positive Histiozyten.

\section{Diskussion}

Die KFD ist eine erstmalig 1972 durch Kikuchi und Fujimoto in Japan beschriebene [2, 3] lymphohistiozytäre Erkrankung bislang unklarer Genese. In asiatischen Ländern endemisch, existieren in Europa nur wenige Fallberichte zu dieser seltenen $\mathrm{Er}$ krankung [4].

Typische, wenngleich unspezifische Symptome dieser Erkrankung sind die persistierende zervikale Lymphadenopathie und Fieber [5]. Das mögliche Auftreten eines unspezifischen Exanthems, von Arthralgien, Fatigue, Organomegalien, Zytopenien, Gewichtsverlust und Nachtschweiß ist unspezifisch und erschwert die differenzialdiagnostische Einordnung [4]. Vor allem die Abgrenzung zu einer malignen Genese der Symptomatik fällt häufig schwer und bedarf einer erfahrenen histopathologischen Beurteilung $[6,7]$.

In einem retrospektiven Review von Kucukardali und Kollegen aus dem Jahre 2007 wurde die Symptomatik von 244 Patienten mit KFD ausgewertet. Hier zeigte sich das Auftreten einer Lymphadenopathie bei $100 \%$ der Patienten. Weitere Symptome waren: Fieber (35\%), Ausschlag (10\%), Arthritis (7\%), Fatigue (7\%) und Hepatosplenomegalie (3\%) [8].

Die Symptome finden sich in ähnlicher Ausprägung auch bei Auftreten der Erkrankung im Erwachsenenalter, wobei sich Fieber und ein Ausschlag häufiger im Kindes- und eine generalisierte Lymphadenopathie häufiger im Erwachsenenalter zeigen [5].

Laborchemisch zeigt sich in der Mehrheit der Patienten ein normwertiges Blutbild [6]. Zytopenien mit unterschiedlicher Ausprägung sind jedoch in der Literatur zu finden [8]. Neben Blutbildveränderungen lässt sich häufig eine erhöhte BSG feststellen [9].

Die Ätiologie dieser typischerweise selbstlimitierenden Erkrankung bleibt weiterhin ungeklärt. Infektiöse Agenzien wie Yersinien, Toxoplasmen, EBV, HHV-6, HHV-8, HTLV-1 und Parvovirus B19 werden als Ursachen diskutiert [10]. Im aktuellen Fall lieBen sich keine Toxoplasmen oder EBV nachweisen. Für die Diagnosefindung der KFD wesentlich ist - neben einer ausführlichen Abklärung infektiologischer Differenzialdiagnosen - eine Lymphknoten-Exstirpation und konsekutive histopathologische Aufarbeitung [11].

Eine spezifische Therapie ist üblicherweise nicht notwendig. Es existieren nur kleine Fallserien, die die Wirksamkeit von Glukokortikoiden nahelegen [12]. Bei persistierendem Fieber oder schlechtem Allgemeinzustand kann die Gabe erwogen werden [13].

Das mögliche Auftreten einer assoziierten hämophagozytischen Lymphohistiozytose $(\mathrm{HLH})$ ist beschrieben und kann den Verlauf dieser Erkrankung deutlich erschweren [4]. Jedoch sind Blutbildveränderungen wie auch ein gesteigertes Ferritin auch unabhängig vom Auftreten einer HLH beschrieben. Hier ist neben der laborchemischen sicher die klinische Präsentation der Patienten entscheidend.

Weiterhin ist auf die Assoziation zu diversen Autoimmunphänomenen (Sjögren-Syndrom, systemischer Lupus erythematodes) zu achten, die auch nach Remission der Erkrankung auftreten können [5]. Eine weiterführende Betreuung durch eine (kinder-)rheumatologische Ambulanz ist sinnvoll, um o.g. assoziierte Erkrankungen frühzeitig zu diagnostizieren und zu behandeln.

\section{FAZIT FÜR DIE PRAXIS}

- Bei Therapie-resistentem Fieber gepaart mit zervikaler Lymphadenopathie sollte auch an die Kikuchi-Fujimoto-Erkrankung (KFD) gedacht werden.

- Bei der KFD handelt es sich um eine Ausschlussdiagnose, die einer histopathologischen Abklärung bedarf.

- Bei schweren Verläufen ist die Gabe von hoch dosierten Glukokortikoiden zu erwägen.

- Die Entwicklung einer HLH oder von Autoimmunphänomenen nach abgelaufener Erkrankung sind möglich und können den Krankheitsverlauf komplizieren.

Interessenkonflikt

Die Autoren geben an, dass kein Interessenkonflikt besteht.

Einhaltung ethischer Richtlinien

Die Autoren bestätigen, dass eine schriftliche Einwilligung der Eltern der Patientin zur Publikation dieses Fallberichts vorliegt.

Autoren

Benedikt Bötticher

Wolfram Klapper ${ }^{2}$

Dirk Klee ${ }^{3}$

Hans-Jürgen Laws ${ }^{1}$

Prasad Thomas Oommen ${ }^{1}$

${ }^{1}$ Heinrich-Heine-Universität Düsseldorf, Medizinische Fakultät, Klinik für KinderOnkologie, -Hämatologie und Klinische Immunologie, Bereich Pädiatrische Rheumatologie, Universitätsklinikum Düsseldorf

2Sektion Hämatopathologie und Lymphknotenregister, Institut für Pathologie, Universitätsklinikum Schleswig-Holstein, Kiel

${ }^{3}$ Institut für Diagnostische und Interventionelle Radiologie, Universitätsklinikum Düsseldorf 


\section{Korrespondenzadresse}

Dr. med. Benedikt Bötticher

Heinrich-Heine Universität Düsseldorf Medizinische Fakultät

Klinik für Kinder-Onkologie, -Hämatologie und Klin. Immunologie Bereich Pädiatrische Rheumatologie Moorenstr. 5, 40225 Düsseldorf Deutschland

Benedikt.Boetticher@med.uni-duesseldorf. de

Literatur

[1] S1-AWMF-Leitlinie Lymphknotenvergrößerung, AWMF-Reg.-Nr. 025-020

[2] Kikuchi M. Lymphadenitis showing focal reticulum cell hyperplasia with nuclear debris and phagocytes: a clinicopathological study. Acta Haematol Jpn 1972(35): 379-380

[3] Fujimoto Y, Kojima Y, Yamaguchi K. Cervical subacute necrotizing lymphadenitis. A
New Clinicopathological Entity 1972(20): 920-927

[4] Lelii M, Senatore L, Amodeo I et al. Kikuchi-Fujimoto disease in children: two case reports and a review of the literature. Italian journal of pediatrics 2018; 44(1): 83

[5] Kim TY, Ha KS, Kim Y et al. Characteristics of Kikuchi-Fujimoto disease in children compared with adults. European journal of pediatrics 2014; 173(1): 111-116

[6] Dorfman RF, Berry G]. Kikuchi's histiocytic necrotizing lymphadenitis: an analysis of 108 cases with emphasis on differential diagnosis. Semin Diagn Pathol 1988; 5(4): 329-345

[7] Escudier A, Courbage S, Meignin V et al. [Kikuchi-Fujimoto disease mimicking malignant lymphoma in adolescents]. Arch Pediatr 2017; 24(5): 471-475

[8] Kucukardali Y, Solmazgul E, Kunter E et al. Kikuchi-Fujimoto Disease: analysis of 244 cases. Clin Rheumatol 2007; 26(1): 50-54

[9] Norris AH, Krasinskas AM, Salhany KE et al. Kikuchi-Fujimoto disease: a benign cause of fever and lymphadenopathy. Am J Med 1996; 101(4): 401-405
[10] Bosch X, Guilabert A, Miquel R et al. Enigmatic Kikuchi-Fujimoto disease: a comprehensive review. American journal of clinical pathology 2004; 122(1): 141-152

[11] Pepe F, Disma S, Teodoro C et al. Kikuchi-Fujimoto disease: a clinicopathologic update. Pathologica 2016; 108(3): 120-129

[12] Jang YJ, Park KH, Seok HJ. Management of Kikuchi's disease using glucocorticoid. J Laryngol Otol 2000; 114(9): 709-711

[13] Payne JH, Evans M, Gerrard MP. Kikuchi-Fujimoto disease: a rare but important cause of lymphadenopathy. Acta paediatrica 2003; 92(2): 261-264

Bibliografie

DOI https://doi.org/10.1055/a-1298-2619 arthritis + rheuma 2020; 40: 449-451 (C) 2020. Thieme. All rights reserved. Georg Thieme Verlag KG, Rüdigerstraße 14, 70469 Stuttgart, Germany ISSN 0176-5167 